\title{
Analytical Assessment to Redesign Effective \& Efficient Servo Gear Assembly Based Solar Sun Tracking System for Solar Parabolic Disc Collectors
}

\author{
Mr. Imran Ali, Hari Kumar Singh, Irfan Ayaz, Shahbaz Alam \\ 1,2,3,4 Department of Energy Engineering, Centre of Excellence, Solar Energy Research \& \\ Utilization, Suresh Gyan Vihar University, Jaipur, India-302025
}

\begin{abstract}
The efficiency of any solar operated system largely depends upon its effective alignment with respect to sunlight and for this there are plethora as of systems available but as per the environmental and geographical circumstances the efficiency of conventional systems vary and because of this the overall output gets deteriorated. There is a strong need to propose a system which is governed directly by the approximated position of sun, calculated by research oriented data. If the system incorporated largely with mechanical system than the dependency of the system defiantly can be trickle down. By analysing that fact into my mind I had opted to redesign solar sun tracking by utilizing total mechanical systems. My endeavour behind the system is to develop and demonstrate a state of the art solar tracking system which is not dependent upon any sensor and can opt its position directly by using approximate solar positioning. The proposed solar sun tracking system comprises of compact reduction gear assembly in which a small DC motor gives its input in low torque and high RPM but by applying reduction gear assembly we are reducing its RPM till 1 in 7 hours so that a highest torque could be generated and the efficient solar sun tracking could become possible.
\end{abstract}

Keywords: alignment, conventional, solar parabolic disc, mechanical, sun tracking, RPM, DC motor, reduction gear assembly, torque, etc.

\section{Introduction:}

Solar sun tracking system is the heart and soul of any solar powered system which harness electricity by concentrating and converting solar sun rays but the efficiency of solar energy production plant is largely depend upon the alignment with the sun.

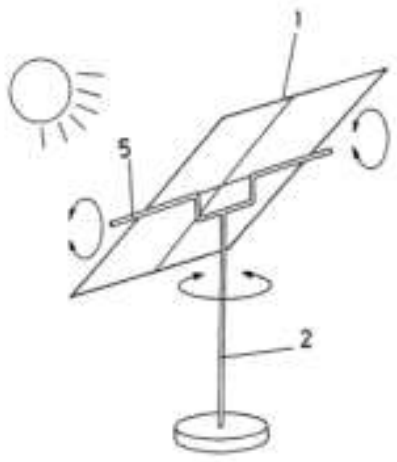

Fig: 1- 2D layout of a conventional solar sun tracker.

There are so many conventional mechanical as well as sensor operated modules are present which act as a solar tracker but there affectivity varies from circumstances to circumstances.

In my design I $\mathrm{m}$ using gear assembly powered solar sun tracking system in which reduction gear assembly is the main constituent which made up of by the combination of spur and helical gears.

The system introduction is as follows-

A) DC carbon brushed motor:- 

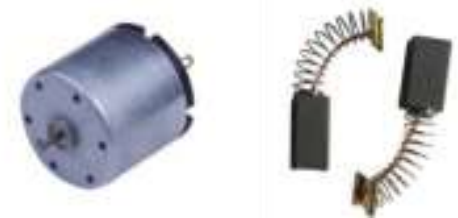

Fig: 2- DC motor and carbon brush.

\section{B) Gear assembly}

In the mechanical solar sun tracking system gear assembly act as a main component as it regulate the rotation provided by DC motor and enhances the torque at the same time.

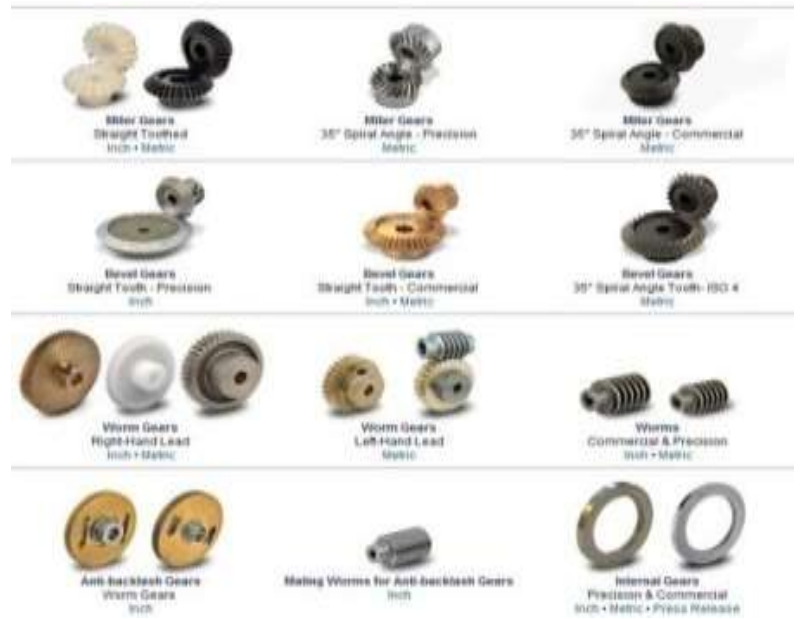

Fig: 3- Different types of gear assemblies

C) Power input assessment system:-

In which we will develop and install a power stabilizing circuit which get power form solar penal itself And it can run independently without any other input.

\section{D) Framing case for solar penal instalment:-}

As per the size and dimensions of the system framing will designed. During my project development, I am designing the solar sun tracker for solar parabolic disc collector of a pre defined dimension.

E) Load capacity assessment: - based upon the hardware.

\section{General introduction of solar sun tracking mechanism:}

Solar powered devices such as solar panels, parabolic disc collectors, etc act efficiently when they are at parallel to the sunrays but keeping them at the line of site of sun all the time is a big challenge and for this solar sun tracker mechanism is applied which provides synchronous momentum with the solar rays and keep the module at the line of site of sun rays all the times. There are plethora of solar sun tracking mechanisms available which having different methodologies and efficiencies accordingly and among them photo-sensor based programmed solar sun tracking mechanism are widely used but due to their inefficiency and high electric consumption made it very much vulnerable to provide failure in the path of smooth functioning. That is why in this paper an ideal mechanical solar sun tracker is being developed.

\section{Literature review:}

The efficient output of every high-concentration solar thermal and parabolic photovoltaic systems is directly proportional to the amount of solar energy acquired by the system, and it is therefore necessary to track the sun's position with a high degree of accuracy. Many systems have been proposed to facilitate this task over the past two decades. Accordingly, this section of the paper commences by providing a high level overview of the sun tracking system field and then describes some of the more significant proposals for closed-loop and open-loop types of sun tracking systems.

If we consider solar sun tracking devices into a chronological order than we found that In 1975, the first 
significant automatic solar tracking systems was presented by McFee, in which an algorithm was developed to compute total received power and flux density distribution in a central receiver solar power system. Than cones the reign of Closed-loop types of sun tracking systems which are generally based on feedback control principles. In these systems, a number of inputs are transferred to a controller from sensors which detect relevant parameters induced by the sun, manipulated in the controller and then yield outputs (i.e. sensor-based).

Themajor achievement in the field of Close loop type solar sun tracker has been achieved and In 1986, In 1998, Khalifa and Al-Mutawalli developed a two-axis sun tracking system to enhance the thermal performance of a compound parabolic concentrator. The system was designed to track the sun's position every three to four minutes in the horizontal plane and every four to five minutes in the vertical plane.

In 2004, Roth et al. designed and constructed a sun tracking system in which a pyrhelio-meter was usedto measure the direct solar radiation. The system was controlled by a closed loop servo system consist of a fourquadrant photo-detector to sense the sun's position and two small DC motors to drive the instrument platform in such a way that the sun's image remained at the center of the four-quadrant detector at all times. Same in this year in 2004, Abdallah and Nijmeh developed an electro-mechanical, two-axis tracking system in which the motion of the sun tracking surface was controlled by an open-loop control algorithm implemented using a PLC unit.

In 2007, Chen et al. presented a sun sensor algorithm based on an analogue optical nonlinear compensation measuring principle.

In a recent study, Grena presented an algorithm for obtaining highly precise values of the solar position. Taking the fractional Universal Time (UT), the date, and the difference between UT and Terrestrial Time (TT) as inputs, the algorithm computed the angular position of the earth with respect to the sun in the ecliptic plane and then used this angle and the inclination angle of the earth's rotational axis to calculate the position of the sun.

\section{Principle:}

To counter the lack of efficiency in current systems available for solar sun tracking, the mechanical way to track the sun is the most convenient and sustainable way and can also be the most preferred way to track heavy objects containing solar power devices such as parabolic solar sun collector, because it acquire a large area and often have bulky size and heavy weight.

The system presented in this research paper is solely based upon mechanical hardware as there is

Any controller or programming is implies. The system tracks sun as according to the time of its movement in the sky. As the sun moves 15 degree in one hour so as the tracker moves the parabolic disc according to it to gets almost into the line of site of sun at every time. The proposed system is driven by a DC motor along with a compact gear assembly which terminate the speed into desired torque and provide regulated momentum which can be controlled directly through input voltage assessment. The speed of revolution remains directly proportional to the voltage input and for this a voltage actuator is also deployed.

\section{Methodology:}

The gear assembly based solar sun tracker contains a specified reduction gear box in such a way so that its ultimate rotation remains in between 160 to 180 degree. The proposed gear box is capable to sustain weight of 47 to $60 \mathrm{~kg}$ solar parabolic disc, it is further used to generate steam from tap water to run a turbine. The gear reduction system consists of a ratio of 1:20:20:20:20:5 gear box which then provides a specific range of rotation if initial input of rotation per minute remains $1000 \mathrm{rpm}$.

\section{Calculation:}

The entire system is depend upon the initial input of fixed rotation which is governed by voltage than if the initial input of DC motor remain 6 volt 1 ampere than-

But, if we evaluate the total time where sunlight remain available than it refers to be approximately 10 Hour

Or, $10 \times 60=6000$ minutes,

But we require total 180 degree displacement of the object than we have to again reduce the rpm through reduction gearbox under the ratio of 1:20:20:20:20:5.

Which are - Initial input of rotation at 12 volt 1 ampere $=1000 \mathrm{rpm}$

First reduction ratio $=1: 20$ than,

$=1000 / 20$

$=50 \mathrm{rpm}$ at first reduction

Second reduction

$=50 / 20=2.5$ 
Third stage of reduction,

$$
=2.5 / 20=0.125
$$

Fourth stage of reduction

$$
=0.125 / 20=0.00625
$$

Fifth stage of reduction

$$
=0.00625 / 5=0.00125
$$

So the final rpm would be -

$=$ Rotation per minute $\times$ total minutes

$=0.00125 \times 600$

$=0.75$ rotation in 600 minutes.

\section{Results:}

As per the calculations and observations it is clear that after implying reduction gear box the total rpm of the motor is $48: 10 \&$ it can be refitted as accordance to the demand to develop a sustainable and reliable solar sun tracker mechanism by minimising the use of electronic circuitry.

The system is further more reliable as it solely powered by renewable electric generated by solar panels.

\section{Future scope:}

As the demand of non conventional energy resources are increasing the total share of solar power in the field of non conventional energy resources are also in increasing order which somehow creating the absolute demand of reliable, sustainable, economic, relatively simple and advance solar sun tracking mechanism which is obvious than the mechanical solar sun tracker could provide a solution to solar sun tracking mechanisms because of its steadfast design and reliability. Also its energy efficient design and non programmed operation give truckload of advantages. In the future, by utilizing the scientific advancement and research the relative hurdles could be easily rectified and after some more amendment in the future it can be one of the most economically viable solar sun tracking mechanism.

\section{Reference}

[1]. Partian, L.D., Solar Cells and their applications (John Wiley \& Sons, New York, 1995).

[2]. Fahrenburch, A. and Bube, R., Fundamentals of Solar Cells (Academic Press, New York, 1983)

Journal Papers:

[3]. E Weise, R Klockner, R Kniel, Ma Sheng Hong, Qin Jian Ping, "Remote Power Supply using Wind and Solar energy - a SinoGerman

[4]. Mechnical Cooperation Project", Beijing International Conference on Wind Energy, Beijing 1995.

[5]. Duryea S, Syed I, Lawrence W, An Automated Battery Management System for Photovoltaic Systems, International Journal of Renewable

[6]. Energy Engineering, Vol 1, No 2, Aug 1999

[7]. Sun Tracking Systems: A Review, Chia-Yen Lee 1, Po-Cheng Chou 2, Che-Ming Chiang 3 and Chiu-Feng Lin 4, Department of Materials Engineering, National Pingtung University of Science and Technology, Pingtung, Taiwan 912; E-Mail: leecy@mail.npust.edu.tw,

[8]. Centre for Resources and Environmental Studies, ANU, Sustainable Energy Systems- Pathways for Australian Energy Reforms

Cambridge University Press, 1994

[9]. IOSR Journal of Mechanical and Civil Engineering (IOSR-JMCE) e-ISSN: 2278-1684, p-ISSN: 2320-334X 